\title{
Hydrogen intercalation of graphene grown on 6H-SiC(0001)
}

\author{
Somsakul Watcharinyanon, Chariya Virojanadara, Jacek Osiecki, A A Zakharov, \\ Rositsa Yakimova, Roger Uhrberg and Leif I Johansson
}

\section{Linköping University Post Print}

N.B.: When citing this work, cite the original article.

Original Publication:

Somsakul Watcharinyanon, Chariya Virojanadara, Jacek Osiecki, A A Zakharov, Rositsa Yakimova, Roger Uhrberg and Leif I Johansson, Hydrogen intercalation of graphene grown on 6H-SiC(0001), 2011, Surface Science, (605), 17-18, 1662-1668.

http://dx.doi.org/10.1016/j.susc.2010.12.018

Copyright: Elsevier http://www.elsevier.com/

Postprint available at: Linköping University Electronic Press http://urn.kb.se/resolve?urn=urn:nbn:se:liu:diva-70327 


\title{
Hydrogen intercalation of graphene grown on $6 \mathrm{H}-\mathrm{SiC}(0001)$
}

\author{
S. Watcharinyanon ${ }^{1}$, C. Virojanadara ${ }^{1}$, J. R. Osiecki ${ }^{1}$, A. A. Zakharov ${ }^{2}$, R. Yakimova ${ }^{1}$, \\ R. I. G. Uhrberg ${ }^{1}$, and L. I. Johansson ${ }^{1}$ \\ ${ }^{1}$ Department of Physics, Chemistry, and Biology, Linköping University, S-58183 \\ Linköping, Sweden \\ ${ }^{2}$ MAX-lab, Lund University, S-22100, Lund, Sweden
}

\begin{abstract}
Atomic hydrogen exposures on a monolayer graphene grown on the $\mathrm{SiC}(0001)$ surface are shown to result in hydrogen intercalation. The hydrogen intercalation induces a transformation of the monolayer graphene and the carbon buffer layer to bi-layer graphene without a buffer layer. The STM, LEED, and core-level photoelectron spectroscopy measurements reveal that hydrogen atoms can go underneath the graphene and the carbon buffer layer and bond to $\mathrm{Si}$ atoms at the substrate interface. This transforms the buffer layer into a second graphene layer. Hydrogen exposure results initially in the formation of bi-layer graphene islands on the surface. With larger atomic hydrogen exposures, the islands grow in size and merge until the surface is fully covered with bi-layer graphene. $\mathrm{A}(\sqrt{3} \times \sqrt{3}) R 30^{\circ}$ periodicity is observed on the bi-layer areas. ARPES and energy filtered XPEEM investigations of the electron band structure confirm that after hydrogenation the single $\pi$-band characteristic of monolayer graphene is replaced by two $\pi$-bands that represent bi-layer graphene. Annealing an intercalated sample, representing bi-layer graphene, to a temperature of $850{ }^{\circ} \mathrm{C}$, or higher, reestablishes the monolayer graphene with a buffer layer on $\mathrm{SiC}(0001)$.
\end{abstract}

Keywords: Epitaxial Graphene, Hydrogen intercalation, Bi-layer, Buffer layer free, Silicon carbide, STM, LEED, Core-level photoelectron spectroscopy, ARPES, Energy filtered XPEEM 


\section{Introduction}

Graphene, a single sheet of $\mathrm{sp}^{2}$-bonded carbon atoms arranged in a honeycomb network, has due to its superior mechanical, electrical, and thermal properties [1] been studied quite extensively for the past few years. The unusual electronic properties, such as a high carrier mobility and insensitivity of the mobility on carrier concentration and temperature, make graphene interesting for electronic applications [2-3]. Graphene can either be prepared by mechanical exfoliation from graphite or by epitaxial growth on different substrates. The former method is known to produce graphene sheets with high structural and electrical qualities [4] but the method is quite delicate and time consuming. The latter, epitaxial growth, is considered an alternative route more suitable for producing graphene wafers for electronic applications. Recently, homogeneous, large area, and high quality monolayer graphene was successfully prepared by epitaxial growth on the $\mathrm{SiC}(0001)$ surface [5-6]. In this process, the $\mathrm{SiC}(0001)$ substrate is heated at a high temperature in an argon atmosphere. At high temperature, $\mathrm{Si}$ atoms desorb and the remaining carbon atoms rearrange and first form a carbon buffer layer at the interface and then graphene layers on top. The buffer layer is comprised of carbon atoms that are covalently bonded to the underlying $\mathrm{SiC}$ substrate and therefore does not show graphitic electronic properties [7]. The buffer layer decouples the graphene layer from the substrate but interactions remain however since the Dirac point is located around $0.4 \mathrm{eV}$ below and not at the Fermi level as in free-standing graphene [7].

Elimination of the carbon buffer layer [8-9] can be one way to produce a more ideal material, i.e., "free standing"-like graphene on the $\mathrm{SiC}$ substrate, for future carbon-based electronic devices. Previous studies [8-9] show that hydrogen exposures can have a pronounced effect on the carbon buffer layer and a model of hydrogen intercalation was suggested. In addition, the hydrogenation mechanism was demonstrated [8-9] to be reversible so a monolayer graphene plus carbon buffer layer sample was transformed to a carbon buffer layer free bi-layer graphene sample and vice versa. In the present work, we do further studies of this hydrogenation mechanism using scanning tunneling microscopy (STM), low energy electron diffraction (LEED), core-level photoelectron spectroscopy (PES), angle resolved photoelectron spectroscopy (ARPES) and X-ray photoelectron 
emission microscopy (XPEEM). An earlier STM study of graphite exposed to atomic hydrogen [10] showed that hydrogen intercalated and was stored between the graphite layers and resulted in the formation of graphene blisters. Recent STM studies of atomic hydrogen exposures on graphene grown on $\operatorname{SiC}(0001)$ [11] and $\operatorname{Ir}(111)$ [12] reported that hydrogen adsorbed on the graphene surface but did not penetrate through the graphene layer into the substrate interface. It was also reported [11] that Si dangling bonds on the $\operatorname{SiC}(0001)(6 \sqrt{3} \times 6 \sqrt{3}) R 30^{\circ}$ surface, i.e. the surface with only the carbon buffer layer, were passivated after exposures if the substrate temperature was kept below ca $400{ }^{\circ} \mathrm{C}$ but not at higher temperatures.

Herein, we report experimental observations of hydrogen intercalation after atomic hydrogen exposures on monolayer graphene prepared epitaxially on $\mathrm{SiC}(0001)$ surfaces. The obtained results support the hydrogenation mechanism suggested in previous studies [8-9]. The changes induced on monolayer graphene by atomic hydrogen exposure were studied using STM and the formation of bi-layer graphene islands was observed when the sample was partly hydrogenated. Larger exposures resulted in full-coverage of bi-layer graphene on the surface. The corrugation and periodicity determined by the underlying $(6 \sqrt{3} \times 6 \sqrt{3}) R 30^{\circ}$ buffer layer structure is removed upon hydrogen intercalation and a flatter surface layer showing a $(\sqrt{3} \times \sqrt{3}) R 30^{\circ}$ periodicity is revealed. Furthermore, LEED and core- level photoelectron spectroscopy results show that the carbon buffer layer is eliminated/transformed after atomic hydrogen exposures and ARPES results demonstrate a clear and distinct effect of hydrogen intercalation on the electronic band structure. We have also applied a new technique, energy filtered XPEEM [13], to image the $\left(\mathrm{E}, \mathrm{k}_{\mathrm{x}}, \mathrm{k}_{\mathrm{y}}\right)$ band structure of monolayer graphene before and after atomic hydrogen exposures.

\section{Experimental methods}

The $n$-type on-axis $6 \mathrm{H}-\mathrm{SiC}(0001)$ substrates were purchased from SiCrystal with chemical and mechanical polishing on the Si face. Before graphene formation, the substrates were cleaned using the RCA method and HF etching in order to remove organic and inorganic contaminations, and surface oxides. The monolayer graphene was 
prepared on the substrates by thermal decomposition of $\mathrm{SiC}$ in an inductively heated furnace under a highly isothermal condition at a temperature of $2000{ }^{\circ} \mathrm{C}$ in an ambient argon pressure of $1 \mathrm{~atm}$ [5]. This method is known to produce homogenous, large area, and high quality graphene on $\mathrm{SiC}(0001)$ [5-6, 14-15]. Some monolayer graphene samples were prepared in situ by direct heating the substrate at a temperature of $1300{ }^{\circ} \mathrm{C}$ under ultra high vacuum conditions.

Hydrogen exposures were performed on the monolayer graphene using an atomic hydrogen source. In the source, a flux of atomic hydrogen $(\mathrm{H})$ was produced from hydrogen molecules $\left(\mathrm{H}_{2}\right)$ that were cracked inside a hot tungsten capillary (about 2000 ${ }^{\circ} \mathrm{C}$ ). During exposure, the samples were annealed at a temperature of $700{ }^{\circ} \mathrm{C}$. An elevated sample temperature was found to be required in order to allow the hydrogen atoms to go underneath the graphene and the buffer layer into the interface [9]. The monolayer graphene samples were exposed to atomic hydrogen at a pressure of about $1 \times 10^{-6} \mathrm{mbar}$. Before and after each hydrogen exposure, the surface structure of the samples was examined by LEED and STM (Omicron VT-STM). The STM was operated at room temperature in a constant current mode with a tunneling current of 0.1-0.5 nA using a $\mathrm{W}$ tip. The samples were also investigated by STM after dehydrogenation.

The dehydrogenation was performed by subsequently annealing the hydrogenated samples at different temperatures, from 750 to $1000^{\circ} \mathrm{C}$. After each annealing step, the depletion of hydrogen was probed by core-level photoelectron spectroscopy and ARPES. These experiments were carried out at MAX-lab in Lund (Sweden) using the beamlines I4 and I311. ARPES measurements were carried out at beamline I4. This beamline is equipped with a spherical grating monochromator and an angular resolved electron energy analyzer with a two-dimensional detector from SPECS. Beamline I311 is equipped with a modified SX-700 monochromator and two experiment stations. The first station consists of a large hemispherical Scienta electron analyzer where core-level photoelectron spectroscopy was performed. $\mathrm{C} 1 \mathrm{~s}$ and $\mathrm{Si} 2 \mathrm{p}$ core-level spectra were acquired at normal emission. Total energy resolutions of $\leq 20 \mathrm{meV}$ at a photon energy of $140 \mathrm{eV}, \leq 100 \mathrm{meV}$ at $450 \mathrm{eV}$, and $\leq 300 \mathrm{meV}$ at $600 \mathrm{eV}$ were selected. The second station is equipped with a spectroscopic photoemission low energy electron microscope (SPELEEM). Energy filtered XPEEM [13] was used to provide direct images of the (E, 
$\mathrm{k}_{\mathrm{x}}, \mathrm{k}_{\mathrm{y}}$ ) electron band structure over the whole first and part of the second Brillouin zone. The base pressure was about $1 \times 10^{-10}$ mbar in all experiment stations.

\section{Results and discussions}

STM images of monolayer graphene grown on $6 \mathrm{H}-\mathrm{SiC}(0001)$ recorded before and after hydrogen exposures are shown in Fig. 1a-c, using a tip bias of $-1 \mathrm{~V}$ and a scan size of $125 \times 125 \mathrm{~nm}^{2}$. A selected part of the corresponding LEED pattern of each state is also shown in Fig. 1d-f. Before hydrogen exposure, the STM image (Fig. 1a) shows large flat terraces of monolayer graphene separated by a step. The selected part of the LEED pattern in Fig. 1d shows one graphene spot (middle) surrounded by six smaller spots. These six spots originate from the $(6 \sqrt{3} \times 6 \sqrt{3}) R 30^{\circ}$ carbon buffer layer underneath the graphene layer. After the first hydrogen exposure for 15 min the STM image in Fig. 1b reveals the presence of islands, all over the surface. The islands are found to cover about $40 \%$ of the total surface area. At this stage the LEED pattern (Fig. 1e) shows that the intensity of the buffer layer spots has decreased considerably. With increasing exposure the islands are found to grow and finally merge and cover the whole surface as illustrated in Fig. 1c. This STM image collected after an additional hydrogen exposure for $15 \mathrm{~min}$. The selected part of the LEED pattern in Fig. 1f now shows an intense graphene spot, while the buffer layer spots have fainted away.

A close-up STM image of an island created after the first hydrogen exposure is shown in Fig. 2a, using a tip bias of $-1 \mathrm{~V}$. This image clearly illustrates the formation of a flatter island compared to the other areas where a larger corrugation is induced on the graphene by the $(6 \sqrt{3} \times 6 \sqrt{3}) R 30^{\circ}$ buffer layer structure [16-17]. The line profile extracted across the island in Fig. 2b demonstrates that the island is flat and that the typical corrugation induced by the buffer layer is not present on the island but on the other areas. Thus, both the STM and LEED results show that the buffer layer is transformed/eliminated initially where the islands are formed and finally on the whole surface after a large enough exposure when the islands have merged and cover the whole surface.

The intercalated samples (with full bi-layer graphene) were then annealed at different temperatures up to $1000{ }^{\circ} \mathrm{C}$. An STM image recorded after such an annealing is shown in Fig. 2c. In comparison to the sample that is partly hydrogenated, Fig. 2a, the image of the annealed sample shows mainly the corrugation and periodicity determined by the 
$(6 \sqrt{3} \times 6 \sqrt{3}) R 30^{\circ}$ buffer layer, which is also indicated by the line profile in Fig. $2 \mathrm{~d}$. The bi-layer areas, i.e., the flat areas with the smaller corrugation, have disappeared. This result indicates the re-establishment of monolayer graphene and the carbon buffer layer after dehydrogenation. However, some small defects, which may arise from leftover hydrogen under the surface or a disorder of the graphene and the buffer layer after transformation from single- to bi-layer and vice versa, are observed on the surface. Further studies on the dehydrogenation utilizing core-level photoelectron spectroscopy and ARPES are presented below.

In order to analyze the surface morphology of the samples before and after hydrogen exposures in greater detail atomically resolved STM images were collected as shown in Fig. 3. The image collected before hydrogen exposure, Fig. 3a, shows a graphene sheet with the honeycomb network that clearly has height variations due to the corrugation of the underlying $(6 \sqrt{3} \times 6 \sqrt{3}) R 30^{\circ}$ buffer layer. The bright areas in the image are related to the protrusions of the buffer layer. A close-up image of the honeycomb structure is shown in Fig. 3b. The distance between the centers of neighboring hexagonal rings is about $0.25 \mathrm{~nm}$ which agrees well with that of the graphene structure $(0.246 \mathrm{~nm})$. After hydrogen exposures, the atomically resolved STM image, Fig. 3c, also shows the honeycomb network but the $(6 \sqrt{3} \times 6 \sqrt{3}) R 30^{\circ}$ superstructure has essentially disappeared.

A magnified STM image after intercalation is displayed in Fig. 3d. Interestingly, besides the honeycomb network of the graphene, a $(\sqrt{3} \times \sqrt{3}) R 30^{\circ}$ periodicity is visible. This is similar to the earlier observation on bi-layer graphene terrace grown on $\mathrm{SiC}(0001)$ in which a $(\sqrt{3} \times \sqrt{3}) R 30^{\circ}$ structure was found in the vicinity of defects (of unknown nature) that were explained to be due to quantum interferences generated by static impurities [18]. The $(\sqrt{3} \times \sqrt{3}) R 30^{\circ}$ periodicity is not visible before the hydrogenation process and is therefore suggested to arise from the bi-layer graphene produced by the hydrogen intercalation.

The effect of the hydrogen exposures on monolayer graphene was also studied by core-level photoelectron spectroscopy. Fig. 4 presents C 1s core-level spectra of monolayer graphene before and after hydrogen exposures and subsequent annealing. The spectra were acquired at a photon energy of $600 \mathrm{eV}$. The $\mathrm{C} 1 \mathrm{~s}$ spectrum of monolayer 
graphene before hydrogen exposure (bottom curve) consists of three components. First, the main emission peak at a binding energy $284.6 \mathrm{eV}$ which is characteristic of the carbon in the graphene sheet. Second, a broader high binding energy component at $285.3 \mathrm{eV}$ corresponding to carbon in the buffer layer, and third, a low binding energy component at $283.6 \mathrm{eV}$ attributed to bulk $\mathrm{SiC}$. The $\mathrm{C}$ 1s spectrum acquired after the small hydrogen exposure shows a decrease of the buffer layer component. A weak component located at $0.6 \mathrm{eV}$ lower binding energy compared to the bulk $\mathrm{SiC}$ component is also discernable. This shifted component is interpreted to originate from the $\mathrm{SiC}$ substrate under the islands formed after a small hydrogen exposure. The intercalated hydrogen forms a dipole layer in-between the graphene and the uppermost Si-C bilayer of the substrate and thereby induces a shift of the substrate $\mathrm{C} 1 \mathrm{~s}$ peak. This was confirmed by the $\mathrm{C} 1 \mathrm{~s}$ data collected on samples that were exposed to larger amounts of hydrogen, so that the sample was fully hydrogenated (see Fig. 4b). Then, only the bulk SiC component shifted $0.6 \mathrm{eV}$ towards lower binding energy was discernable, and the buffer layer component had essentially disappeared. Also these C 1s results thus suggest that atomic hydrogen do intercalate in-between the buffer layer and the substrate.

The dehydrogenation was studied by subsequently annealing the intercalated samples at temperatures from 750 to $1000{ }^{\circ} \mathrm{C}$. Core-level spectra were recorded at each annealing step and a series of such C 1s spectra is also presented in Fig 4. Annealing the hydrogenated sample at $750{ }^{\circ} \mathrm{C}$ for a few minutes did not have a significant effect. The spectrum shows the same contributions from graphene, buffer layer and bulk $\mathrm{SiC}$ that do and do not interact with the hydrogen, as before the anneal. Only small changes such as a sharpening of the graphene peak and a slight broadening of the buffer layer component were observed compared to that of the hydrogenated sample before annealing. After annealing the sample at $850{ }^{\circ} \mathrm{C}$ for a few minutes, the bulk $\mathrm{SiC}$ component, shifted to 0.6 $\mathrm{eV}$ lower binding energy has essentially disappeared. The intensity of the buffer layer component is significantly increased while the intensity of the graphene component is slightly decreased. This indicates the temperature at which hydrogen desorption starts, which results in that the second graphene layer is transformed back to a carbon buffer layer. After annealing the sample at $950{ }^{\circ} \mathrm{C}$ and $1000{ }^{\circ} \mathrm{C}$, the shape of $\mathrm{C} 1 \mathrm{~s}$ spectrum is 
restored and looks similar to the one from monolayer graphene before hydrogen exposures.

A similar trend is observed in acquired Si $2 p$ spectra, as shown in Fig. 5. The Si $2 p$ spectrum collected from monolayer graphene shows a bulk $\mathrm{SiC}$ signal at a $\mathrm{Si} 2 \mathrm{p}_{3 / 2}$ binding energy of $101.3 \mathrm{eV}$. After a small hydrogen exposure, a shoulder is observed at low binding energy. The shoulder corresponds to a Si $2 \mathrm{p}$ component shifted about $0.6 \mathrm{eV}$ towards lower binding energy compared to the original bulk component. The shift and relative intensity of this component are thus similar to the observations made for the shifted bulk component in the $\mathrm{C} 1 \mathrm{~s}$ spectrum. The shoulder is interpreted to originate from the interaction of hydrogen and silicon atoms at the graphene-SiC interface resulting in $\mathrm{Si}-\mathrm{H}$ bonds at the intercalated islands. The intensity of the shoulder increases somewhat after annealing the sample at $750{ }^{\circ} \mathrm{C}$ but then decrease dramatically when the annealing temperature is increased to $850^{\circ} \mathrm{C}$. No significant change was observed when increasing the annealing temperature up to 950 and $1000{ }^{\circ} \mathrm{C}$. These core-level photoelectron spectroscopy results indicate that the atomic hydrogen can go underneath the graphene and the buffer layer and bond to $\mathrm{Si}$ atoms in the substrate. Moreover, the series of $\mathrm{C} 1 \mathrm{~s}$ and $\mathrm{Si} 2 \mathrm{p}$ spectra show that the hydrogen intercalation of graphene is reversible, i.e., the graphene layer that is created by hydrogen exposure transforms back to the carbon buffer layer upon heating the intercalated sample at a temperature of about $850{ }^{\circ} \mathrm{C}$ or higher.

Further evidence of the hydrogen-intercalation process was obtained by investigations of the band structure. ARPES was utilized to investigate the effect of hydrogenation on the electron band structure of graphene around the $\bar{K}$ point in the hexagonal Brilloun zone. Results are shown in Figs. 6a and b, which were collected along the $\bar{K} \rightarrow \bar{\Gamma}$ direction. The number of graphene layers present on the $\operatorname{SiC}(0001)$ surface can be identified by the number of $\pi$-band branches [19] at the $\bar{K}$ point. The band structure of monolayer graphene shows a single $\pi$-band around the $\bar{K}$ point as seen in Fig. 6a. Unlike the free-standing graphene, the Dirac point of monolayer graphene on $\mathrm{SiC}(0001)$ is located around $0.4 \mathrm{eV}$ below and not at the Fermi level due to the substrate interactions [7]. After hydrogen exposures, see Fig. 6b, we observe two $\pi$-bands, similar to the calculated band structure of bi-layer graphene [20], showing that the monolayer graphene 
with the buffer layer underneath transform to bi-layer graphene due to hydrogen intercalation. Moreover, the shift of the Dirac point towards the Fermi level upon hydrogenation indicates that hydrogen intercalation gives rise to a weakening of the graphene substrate interactions. Two-dimensional momentum resolved photoelectron angular distribution patterns collected using energy filtered XPEEM [13] show the same band structure result but in a different way. The direct image of $\left(\mathrm{E}, \mathrm{k}_{\mathrm{x}}, \mathrm{k}_{\mathrm{y}}\right)$ band structure over the whole Brillouin zone is obtained. For the initial monolayer graphene sample the shape of the well known graphene band structure with the Dirac points approximately 0.4 $\mathrm{eV}$ below the Fermi level is clearly reproduced, in Figs. 6d-f. After atomic hydrogen exposure the second $\pi$-band in the electronic band structure becomes clearly observable in Figs. 6g-i. The two $\pi$-bands are most apparent in the angular distribution patterns collected at 2.0 and $2.5 \mathrm{eV}$ below the Fermi level, respectively, and not the Dirac point. This we believe to be mainly due to that the relative intensity of the lower lying energy band is so much higher than that of the other band at $0.4-0.6 \mathrm{eV}$ below the Fermi level compared to 2.0 and $2.5 \mathrm{eV}$. The evolution of the $\pi$-band with annealing temperature was also investigated using ARPES. At $750{ }^{\circ} \mathrm{C}$ no changes were observed but when increasing the temperature to $850{ }^{\circ} \mathrm{C}$ the upper $\pi$-band became considerably fainter and from $900{ }^{\circ} \mathrm{C}$ to $1000{ }^{\circ} \mathrm{C}$ only one band remained, as in Fig. 6a. This indicates that the monolayer graphene is re-established after dehydrogenation.

\section{Summary}

Using different surface characterization techniques we demonstrate that after exposure to atomic hydrogen monolayer graphene and carbon buffer layer on $\mathrm{SiC}(0001)$ transform to buffer layer free bi-layer graphene. Atomic hydrogen exposures are shown to result in hydrogen intercalation. In the intercalation process, hydrogen atoms penetrate through the graphene and buffer layer and bond to $\mathrm{Si}$ atoms in the substrate, as observed in core-level photoelectron spectroscopy measurements. The buffer layer is lifted up and transformed into a second graphene layer contributing to the initial formation of islands on the surface as observed in STM images. A suppression of the characteristic features from the buffer layer in the LEED pattern, and atomic resolution STM images, was distinguished after initial hydrogen exposures. With larger exposure, the islands were found to grow in size 
and merge until the surface was fully covered with bi-layer graphene. ARPES and energy dispersive XPEEM measurements indicated a clear and distinct effect on the electronic band structure upon hydrogen intercalation. The changes observed in the $\pi$-valence band showed clearly a transformation from monolayer to bi-layer graphene upon hydrogenation.

The intercalation was observed to be reversible. The monolayer graphene with buffer layer could be re-established by heating the hydrogen intercalated sample to a temperature higher than ca. $850{ }^{\circ} \mathrm{C}$. Our findings demonstrate that through hydrogen intercalation, the carbon buffer layer, that is considered to be a main obstacle for the development of graphene- based electronic devices, can be eliminated. Moreover, by hydrogen intercalation, the electronic properties of the graphene grown on $\mathrm{SiC}(0001)$ can be tailored. If the hydrogen intercalation improves the electron and hole mobilities is of interest for electronic applications. Hydrogen intercalation provides $p$-doping [8, 21] while Li intercalation [22] provides strong $n$-doping. This could possibly be exploited to producing $p-n$ junctions using graphene grown on $\mathrm{SiC}$ substrates.

\section{Acknowledgments}

This work is supported by grants from the Swedish National Energy Administration. We would like to thank the MAX-lab staffs for assistance during the synchrotron-based experiments.

\section{References}

[1] A.K. Geim, Science 324 (2009) 1530.

[2] C. Berger, Z. Song, T. Li, X. Li, A.Y. Ogbazghi, R. Feng, Z. Dai, A.N. Marchenkov, E.H. Conrad, P.N. First, W.A. de Heer, J. Phys. Chem. B 108 (2004) 19912.

[3] A.K. Geim, K.S. Novoselov, Nat. Mater. 6 (2007) 183.

[4] K.S. Novoselov, A.K. Geim, S.V. Morozov, D. Jiang, Y. Zhang, S.V. Dubonos, I.V. Grigorieva, A.A. Firsov, Science 306 (2004) 666.

[5] C. Virojanadara, M. Syväjärvi, R. Yakimova, L.I. Johansson, A.A. Zakharov, T. Balasubramanian, Phys. Rev. B 78 (2008) 245403. 
[6] R. Yakimova, C. Virojanadara, D. Gogova, M. Syväjärvi, D. Siche, K. Larsson, L.I. Johansson, Mater. Sci. Forum. 645-648 (2010) 565.

[7] K.V. Emtsev, F. Speck, T. Seyller, L. Ley, J.D. Riley, Phys. Rev. B 77 (2008) 155303.

[8] C. Riedl, C. Coletti, T. Iwasaki, A.A. Zakharov, U. Starke, Phys. Rev. Lett. 103 (2009) 246804.

[9] C. Virojanadara, A.A. Zakharov, R. Yakimova, L.I. Johansson, Surf. Sci. 604 (2010) L4.

[10] Z. Waqar, J. Mater. Sci. 42 (2007) 1169.

[11] N.P. Guisinger, G.M. Rutter, J.N. Crain, P.N. First, J.A. Stroscio, Nano Lett. 9 (2009) 1462.

[12] R. Balog, B. Jorgensen, L. Nilsson, M. Andersen, E. Rienks, M. Bianchi, M. Fanetti, E. Laegsgaard, A. Baraldi, S. Lizzit, Z. Sljivancanin, F. Besenbacher, B. Hammer, T.G. Pedersen, P. Hofmann, L. Hornekaer, Nat. Mater. 9 (2010) 315.

[13] A.A. Zakharov, C. Virojanadara, S. Watcharinyanon, R. Yakimova, L.I. Johansson, To be published.

[14] K.V. Emtsev, A. Bostwick, K. Horn, J. Jobst, G.L. Kellogg, L. Ley, J.L. McChesney, T. Ohta, S.A. Reshanov, J. Röhrl, E. Rotenberg, A.K. Schmid, D. Waldmann, H.B. Weber, T. Seyller, Nat. Mater. 8 (2009) 203.

[15] C. Virojanadara, R. Yakimova, J.R. Osiecki, M. Syväjärvi, R.I.G. Uhrberg, L.I. Johansson, A.A. Zakharov, Surf. Sci. 603 (2009) L87.

[16] G.M. Rutter, N.P. Guisinger, J.N. Crain, E.A.A. Jarvis, M.D. Stiles, T. Li, P.N. First, J.A. Stroscio, Phys. Rev. B 76 (2007) 235416.

[17] P. Lauffer, K.V. Emtsev, R. Graupner, T. Seyller, L. Ley, Phys. Rev. B 77 (2008) 155426.

[18] P. Mallet, F. Varchon, C. Naud, L. Magaud, C. Berger, J.-Y. Veuillen, Phys. Rev. B 76 (2007) 041403.

[19] T. Ohta, A. Bostwick, J.L. McChesney, T. Seyller, K. Horn, E. Rotenberg, Phys. Rev. Lett. 98 (2007).

[20] A. Bostwick, H. Ohta, J.L. McChesney, K.V. Emtsev, T. Seyller, K. Horn, E. Rotenberg, New J. Phys. 9 (2007) 385. 
[21] F. Speck, M. Ostler, J. Röhrl, J. Jobst, D. Waldmann, M. Hundhausen, L. Ley, H.B. Weber, T. Seyller, Mater. Sci. Forum. 645 - 648 (2009) 629.

[22] C. Virojanadara, S. Watcharinyanon, A.A. Zakharov, L.I. Johansson, Phys. Rev. B 82 (2010) 205402. 


\section{Figure captions}

Fig. 1. STM images collected at $V=-1 \mathrm{~V}$ and $\mathrm{I}=500 \mathrm{pA}$ of a) monolayer graphene, $b$ ) after a small hydrogen exposure, and c) after a large hydrogen exposure. d) Selected part of the LEED pattern collected at $\mathrm{E}=107 \mathrm{eV}$ from monolayer graphene, e) after a small hydrogen exposure, and f) after a large hydrogen exposure."

Fig. 2. STM images of a) an island created by the hydrogen exposure $(V=-1 V, I=$ $500 \mathrm{pA}), \mathrm{b})$ line profile across the island, c) a dehydrogenated sample showing mainly $(6 \sqrt{3} \times 6 \sqrt{3}) R 30^{\circ}$ structure from the buffer layer $(\mathrm{V}=-2 \mathrm{~V}, \mathrm{I}=100 \mathrm{pA})$, and $\left.\mathrm{d}\right)$ line profile across the $(6 \sqrt{3} \times 6 \sqrt{3}) R 30^{\circ}$ structure.

Fig. 3. Atomically resolved STM images of a) and b) monolayer graphene (V = -0.5V, I $=200 \mathrm{pA})$, and $\mathrm{c})$ and $\mathrm{d})$ after hydrogenation $(\mathrm{V}=-0.1 \mathrm{~V}, \mathrm{I}=100 \mathrm{pA})$. The white dashed diamonds indicates the $(\sqrt{3} \times \sqrt{3}) R 30^{\circ}$ periodicity.

Fig. 4. Normalized C 1s core level spectra of a) monolayer graphene before and after hydrogenation and subsequent annealing at 750, 850, 950, $1000{ }^{\circ} \mathrm{C}$. b) Fully hydrogenated graphene along with monolayer graphene before hydrogenation. The spectra were acquired at a photon energy of $600 \mathrm{eV}$.

Fig. 5. Normalized Si $2 p$ core level spectra of monolayer graphene before and after hydrogenation and subsequent annealing at $750,850,950,1000{ }^{\circ} \mathrm{C}$. The spectra were acquired at a photon energy of $140 \mathrm{eV}$.

Fig. 6. $\pi$-band dispersion around the $\bar{K}$-point of a) monolayer graphene, and b) after hydrogenation. c) Schematic drawing of the 2D Brillouin zone of graphene. The arrow indicates the measured direction around the $\bar{K}$ point. d-f) The two-dimensional momentum resolved photoelectron angular distribution patterns of monolayer graphene, and g-i) after hydrogenation. 

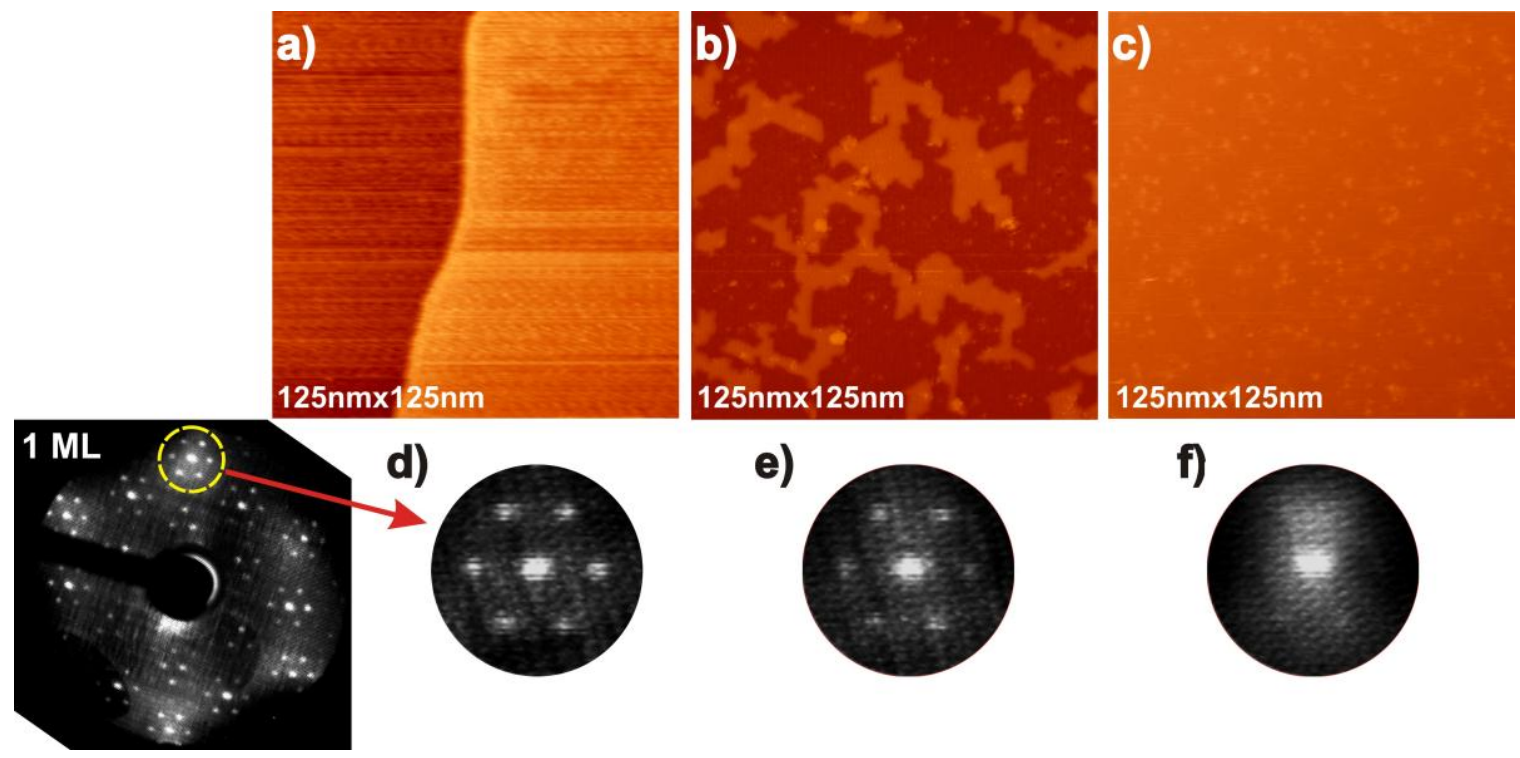

e)
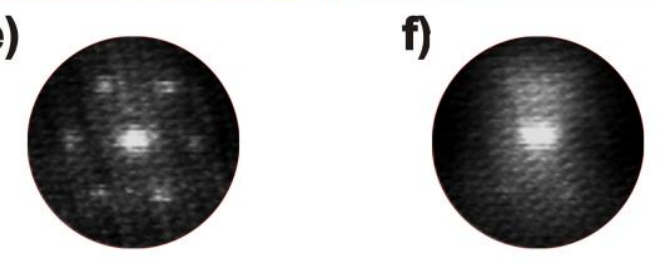

Fig. 1 

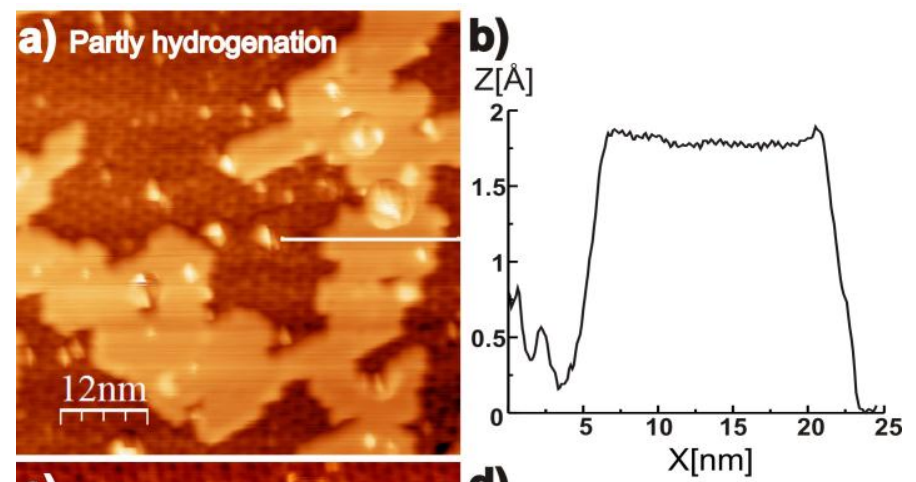

C) After heated hydrogen away
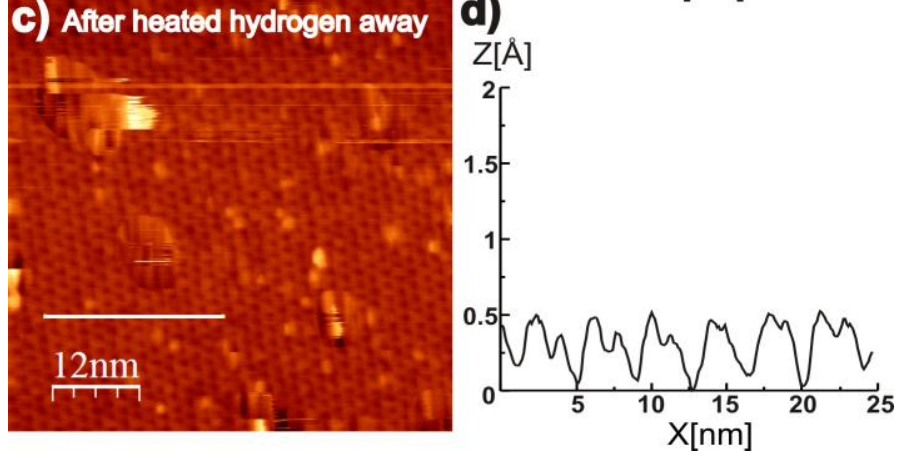

Fig. 2 

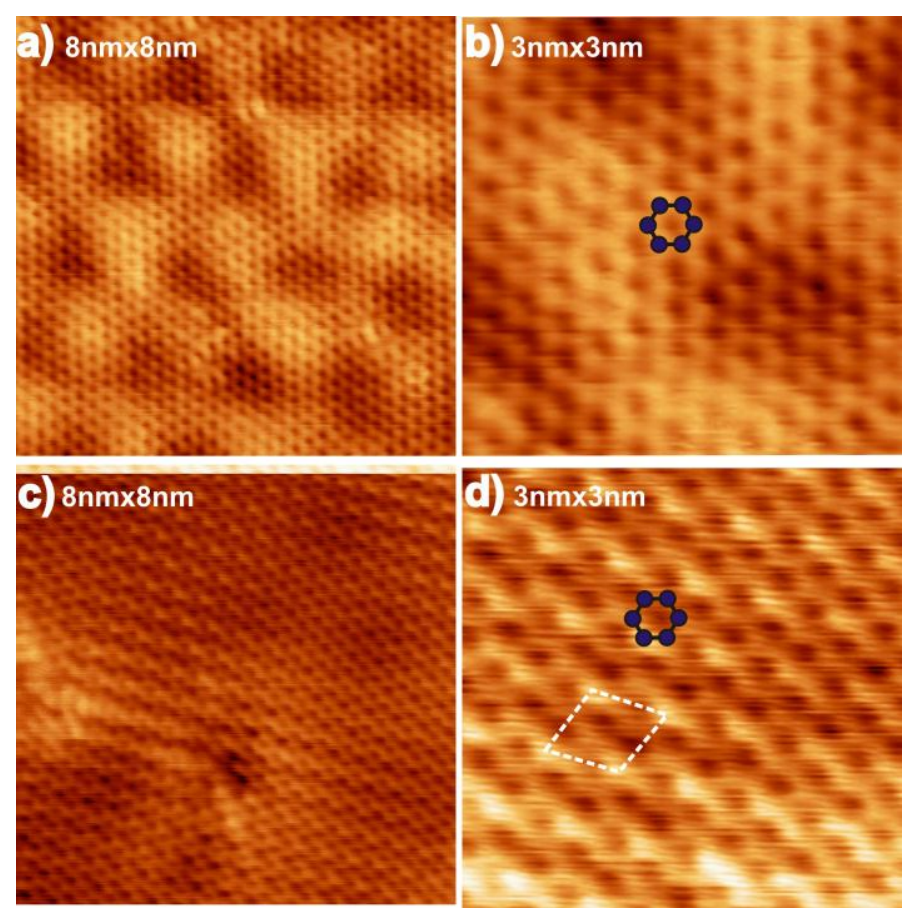

Fig. 3 


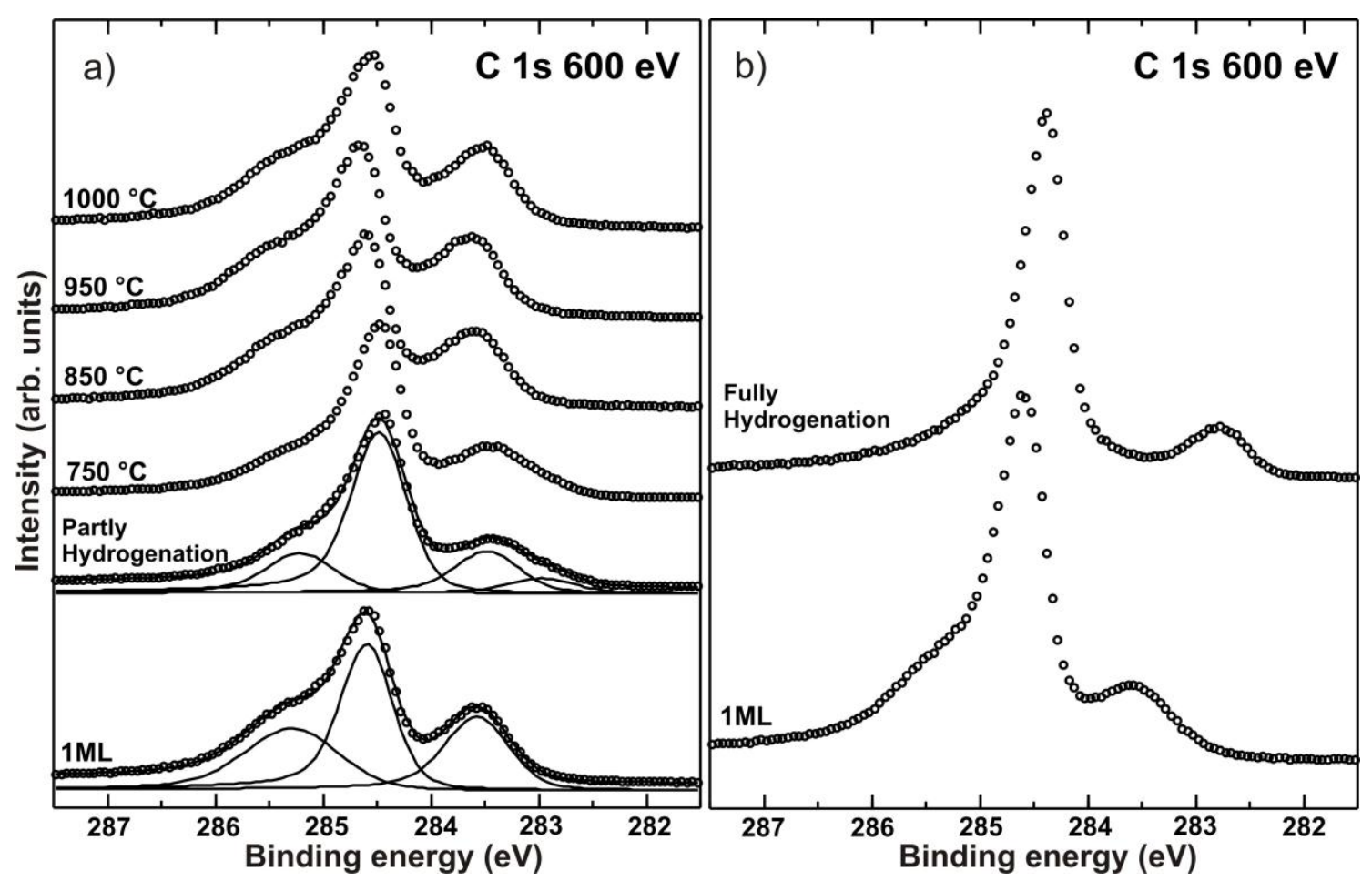

Fig. 4 


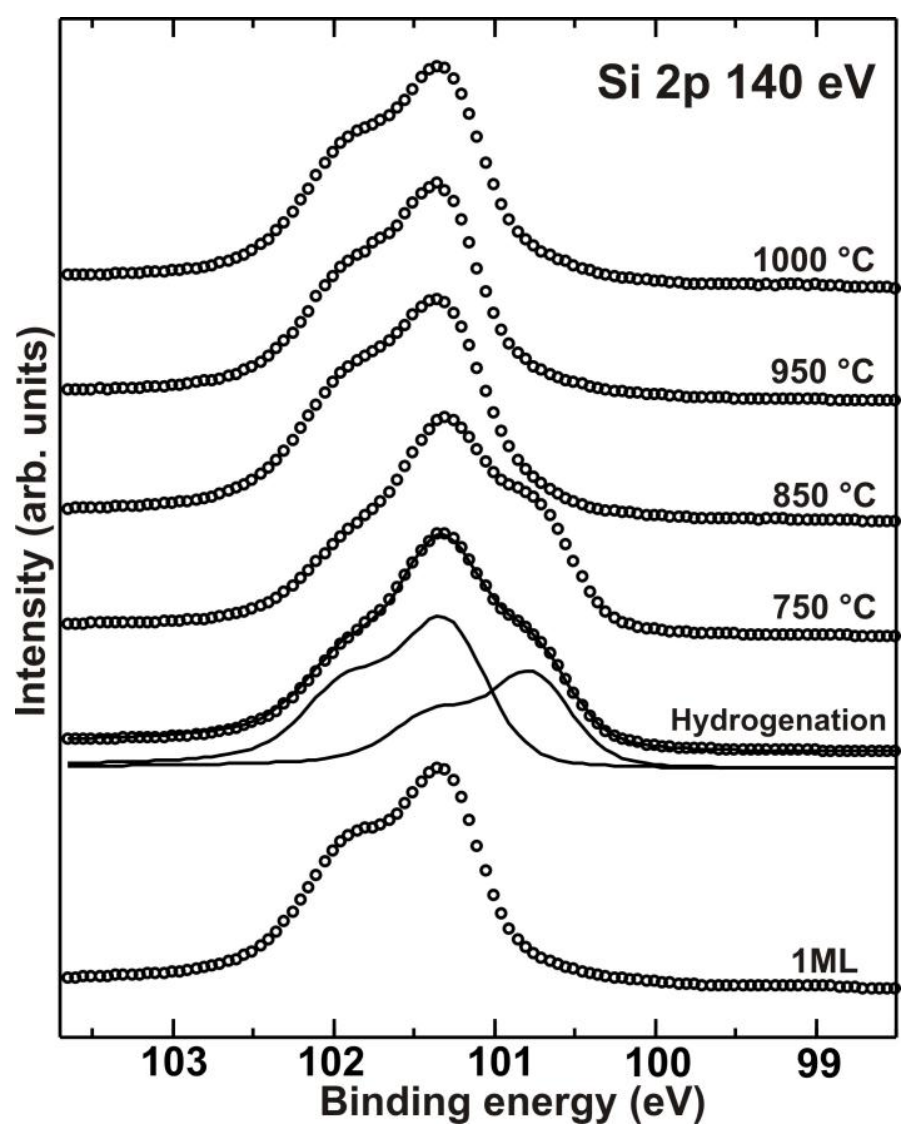

Fig.5 
1ML graphene

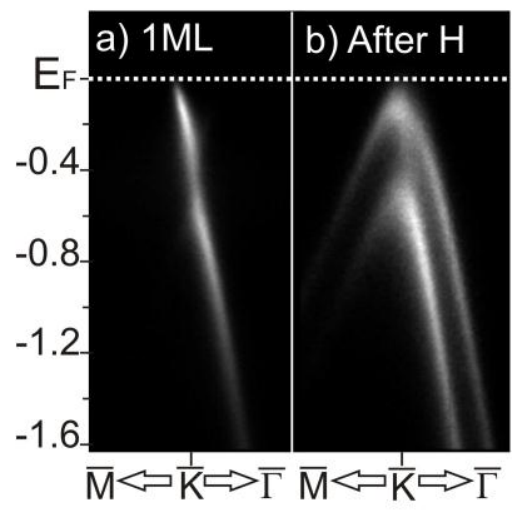

c)
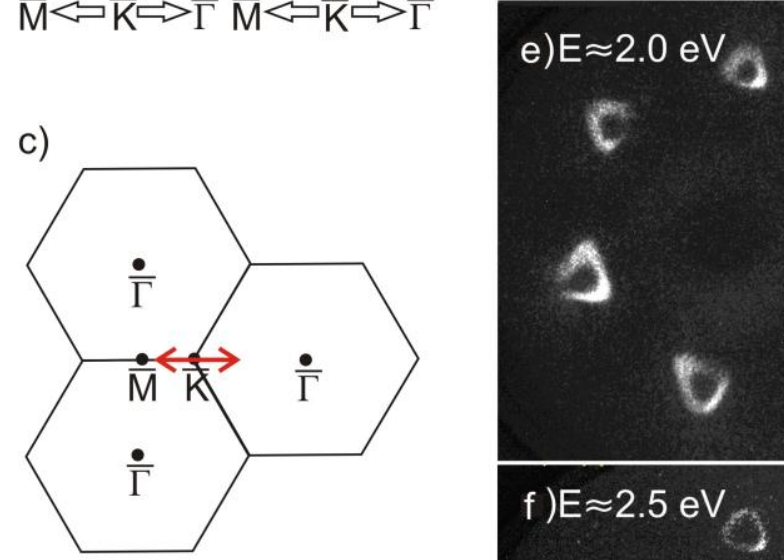

s

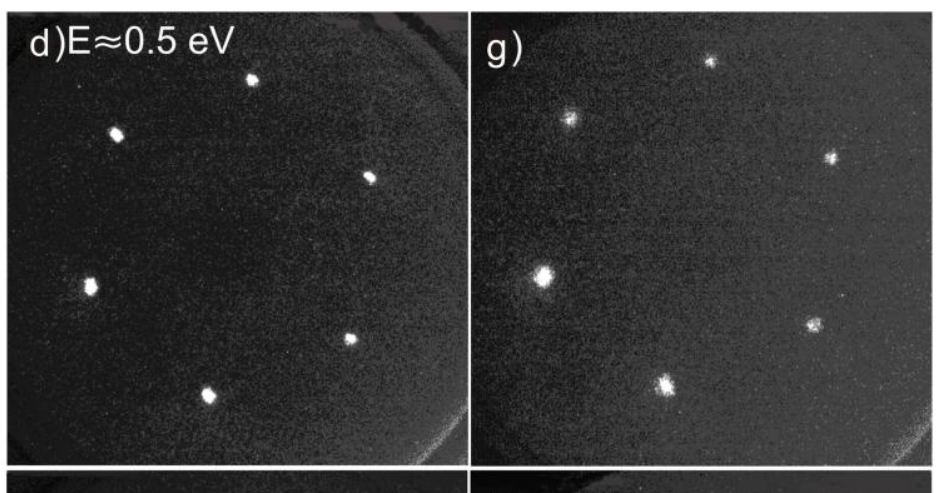

h)

9
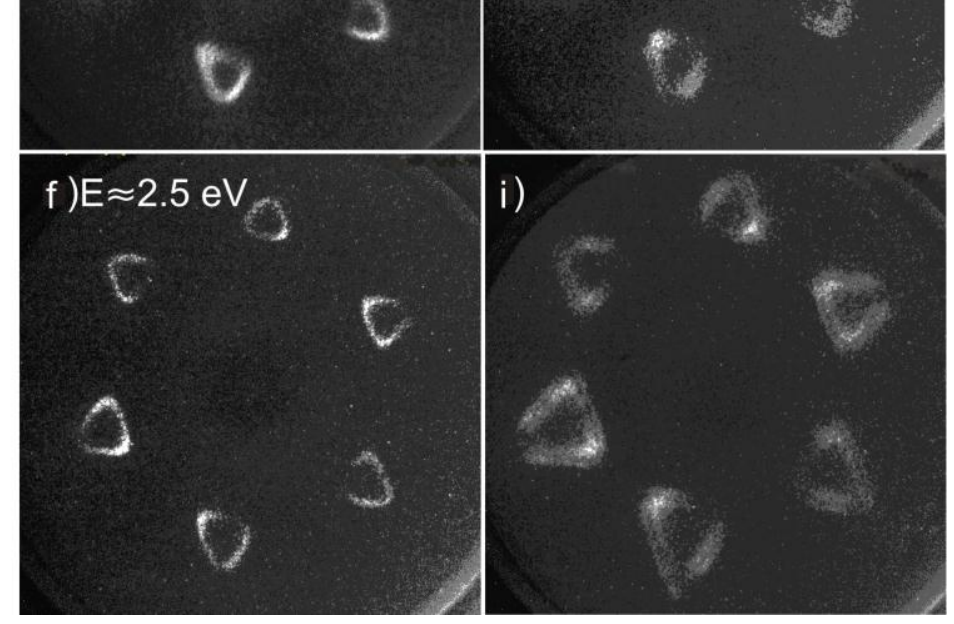

Fig.6 